\title{
Effect of the contraction ratio upon viscoelastic fluid flow in three-dimensional square-square contractions
}

\author{
P.C. Sousa ${ }^{\text {a }}$, P.M. Coelho ${ }^{\text {b }}$, M.S.N. Oliveira ${ }^{a}$, M.A. Alves ${ }^{\mathrm{a}, *}$ \\ a Departamento de Engenharia Química, CEFT, Faculdade de Engenharia da Universidade do Porto, Rua Dr. Roberto Frias, $4200-465$ Porto, Portugal \\ ${ }^{\mathrm{b}}$ Departamento de Engenharia Mecânica, CEFT, Faculdade de Engenharia da Universidade do Porto, Rua Dr. Roberto Frias, $4200-465$ Porto, Portugal
}

\section{A R T I C L E I N F O}

\section{Article history:}

Received 17 June 2010

Received in revised form

5 December 2010

Accepted 10 December 2010

Available online 16 December 2010

Keywords:

Visualization

Viscoelasticity

Non-Newtonian fluids

Laminar flow

Particle Image Velocimetry

3D contraction flow

\begin{abstract}
A B S T R A C T
In this work we investigate the laminar flow through square-square sudden contractions with various contraction ratios $(C R=2.4,4,8$ and 12$)$, using a Newtonian fluid and a shear-thinning viscoelastic fluid. Visualizations of the flow patterns were carried out using streak line photography and detailed velocity field measurements were performed using particle image velocimetry. The experimental results are compared with numerical predictions obtained using a finite-volume method. For the Newtonian fluid, a corner vortex is found upstream of the contraction and increasing flow inertia leads to a reduction of the vortex size. Good agreement is observed between experiments and numerical simulations. For the shearthinning fluid flow a corner vortex is also observed upstream of the contraction independently of the contraction ratio. Increasing the elasticity of the flow, while still maintaining low inertia flow conditions, leads to a strong increase of the vortex size, until an elastic instability sets in and the flow becomes timedependent at $D e \approx 200,300,70$ and 450 for $C R=2.4,4,8$ and 12, respectively. At low contraction ratios, viscoelasticity brings out an anomalous divergent flow upstream of the contraction. For both fluids studied the flow presents a complex three-dimensional helical vortex structure which is well predicted by numerical simulations. However, for the viscoelastic fluid flow the maximum Deborah number achieved in the numerical simulations is about one order of magnitude lower than the critical Deborah number for the onset of the elastic instability found in the experiments.
\end{abstract}

(c) 2010 Elsevier Ltd. All rights reserved.

\section{Introduction}

Non-Newtonian fluids have been extensively studied since the 1940s, when investigations carried out during the Second World War showed several interesting phenomena and stimulated numerous industrial applications (Denn, 2004). Thereafter, flows of viscoelastic fluids and consequently the impact of their rheological properties on the flow patterns were the aim of several research studies. In this context, special attention was given to entry-flow problems, and in particular to contraction flows (e.g. Cable and Boger, 1978a, b, 1979; White and Baird, 1988; Quinzani et al., 1995; Rothstein and McKinley, 1999; Alves et al., 2004; Lu et al., 2006; Oliveira et al., 2007; Walters et al., 2009; Chung et al., 2009).

Several authors have investigated the Newtonian and non-Newtonian fluid flow through sudden contractions with circular and planar geometrical configurations, with particular emphasis on the understanding of the flow patterns developed near the re-entrant and salient corners (e.g. Boger et al., 1986; Nigen and Walters, 2002).

\footnotetext{
* Corresponding author. Tel.: +351 225081680; fax: +351225081449.

E-mail addresses: psousa@fe.up.pt (P.C. Sousa),pmc@fe.up.pt (P.M. Coelho), monica.oliveira@fe.up.pt (M.S.N. Oliveira),mmalves@fe.up.pt (M.A. Alves).
}

As described by Boger (1987) in his review, viscoelastic fluid flow through axisymmetric contractions presents a rich variety of flow patterns which depend on the contraction ratio, the fluid rheology and the flow conditions. For instance, large corner vortices develop upstream of the contraction plane, which are normally preceded by the formation of lip vortices near the re-entrant corner, as the Deborah number increases. In order to investigate the influence of inertial effects on the flow of non-Newtonian fluids through axisymmetric contractions, Cable and Boger (1978a, b, 1979) analyzed the flow of aqueous solutions of polyacrylamide (PAA) in 2:1 and 4:1 axisymmetric contractions. At low flow rates, i.e. in the absence of significant inertial and elastic effects, the flow behavior is similar to that found for a Newtonian fluid. As the flow rate is increased, two different flow regimes were found, namely the vortex growth regime and the onset of diverging streamlines. Evans and Walters (1986, 1989) and Walters and Webster (1982), amongst others, also studied experimentally the flow of PAA solutions through planar contractions. Later, Purnode and Crochet (1996) simulated numerically the experimental results obtained previously by Evans and Walters $(1986,1989)$ using the FENE-P constitutive equation. The effect of the polymer concentration, the contraction ratio and the shape of the re-entrant corner on the appearance and enhancement of the vortices were established. The authors found that the observed corner vortices increase in strength with polymer concentration and a lip vortex appears for 
low-viscosity solutions. Additionally, the lip vortex sets in for the contraction ratio of 4 in the presence of inertial effects, while for higher contraction ratios inertia is not essential.

Most of the previous numerical works focused on the development of robust and stable numerical methods to simulate the flow of complex fluids with viscoelastic behavior, usually described using a differential constitutive equation. Furthermore, two-dimensional (2D) steady-state numerical simulations have been more exploited than three-dimensional (3D) ones, primarily due to the limited computer resources. However, the non-Newtonian fluid flow in 3D geometries leads, in some cases, to a complex and time-dependent flow behavior and therefore only time-dependent 3D numerical calculations can accurately predict the characteristics of the flow. Most of the 3D viscoelastic fluid flows investigated numerically are for planar contractions with ending walls separated by a finite distance, usually defining a large aspect ratio. For example, in the work of Mompean and Deville (1997) the flow of an Oldroyd-B fluid was simulated numerically using $2 \mathrm{D}$ and quasi-2D calculations. The authors found that the results obtained from 3D calculations are substantially different from those obtained assuming a 2D approximation. Xue et al. (1998) also studied the viscoelastic fluid flow in the benchmark 4:1 planar contraction, using a three-dimensional finite-volume method. The 3D results were also compared with 2D calculations using the upper-convected Maxwell (UCM) and the Phan-Thien-Tanner (PTT) models, in order to describe Boger and shear-thinning fluids, respectively, and the 2D simulations were a good representation of the flow.

For the numerical simulation of viscoelastic fluid flow, it is very important to select an adequate constitutive equation and the corresponding rheological parameters in order to reproduce accurately the shear viscosity, normal stress and extensional properties, since these material functions have a direct impact on the flow behavior.

The square-square contraction is a simple geometrical arrangement that leads to complex 3D flow patterns which can be useful as a benchmark solution for the development of 3D numerical codes in computational rheology. Sirakov et al. (2005) studied the flow of a viscoelastic fluid from a square channel to a circular pipe with smaller cross-sectional area. The authors reported the formation of an open vortical structure in the flow through the contraction. This three-dimensional behavior observed experimentally was successfully captured in the numerical results obtained using the eXtended Pom-Pom model. Alves et al. (2005) investigated the Newtonian and non-Newtonian fluid flow through a 4:1 square-square contraction. Two different Newtonian fluids were used in the experiments and the corresponding flow patterns were simulated numerically. Moreover, the flow of a viscoelastic fluid with a nearly constant shear viscosity (Boger fluid) was also studied experimentally. More recently, Sousa et al. (2009) investigated the effect of the contraction ratio on the flow through square-square contractions of a Newtonian fluid and the Boger fluid used by Alves et al. (2005). Contraction ratios of $2.4,4,8$ and 12 were explored and for the Newtonian fluid the corner vortex formed in the upstream channel was found to decrease in size monotonically as the Reynolds number increases. On the other hand, the viscoelastic fluid presented a different flow behavior that depends strongly on the contraction ratio. For the lower contraction ratios, diverging streamlines upstream of the contraction plane were observed with an initial decrease in the vortex size followed by an increase as the Deborah number (or the flow rate) increases. For the higher contraction ratios, vortex enhancement was found with localized diverging streamlines occurring near the re-entrant corner. Furthermore, at high Deborah number flows, an elastic instability sets in and the flow eventually becomes time-dependent. The flow through 3D square-square contractions using a viscoelastic fluid with a shearthinning rheological behavior was also investigated experimentally and numerically by Alves et al. (2008) using a 4:1 contraction ratio. The authors found a strong vortex enhancement, with the flow eventually becoming unsteady as the Deborah number increases. The correct representation of the extensional behavior of the fluid was shown to be important in order to obtain accurate flow predictions.

In this work, we investigate the flow of a shear-thinning fluid through 3D square-square contractions in more detail and assess the influence of the contraction ratio upon the flow patterns and the velocity field. For that purpose, we show the flow patterns visualized using streak line photography and quantify the vortex activity in terms of the vortex length. Moreover, we present the velocity field obtained from particle image velocimetry (PIV) measurements. The experiments were performed with a Newtonian and a shear-thinning viscoelastic fluid and numerical simulations of the flow were performed using a finite volume method (Oliveira et al., 1998). The PTT model (Phan-Thien and Tanner, 1977) was used to describe the rheology of the shear-thinning viscoelastic fluid.

The remainder of this paper is organized as follows: in Section 2 the experimental set-up is described, as well as the experimental techniques used in the characterization of the flow. The fluids used and the rheological characterization is presented in Section 3. The numerical method employed for the simulation of the Newtonian and non-Newtonian fluid flow as well as the characteristics of the computational meshes used are described in Section 4. In Sections 5 and 6 we present and discuss the results regarding the flow patterns and velocity field, respectively. Finally, in Section 7 the main conclusions are summarized.

\section{Experimental set-up and techniques}

The test rig was made of transparent acrylic and placed in a dark room for flow visualizations and particle image velocimetry measurements. Fig. 1 shows schematically the experimental set-up.

The main duct is composed of two parts: an upstream channel with a fixed width of $2 \mathrm{H}_{1}=24 \mathrm{~mm}$ and an interchangeable square duct with a smaller inner side length, which fits precisely in the larger square duct. To assess the effect of the contraction ratio, this downstream side length can be set to $2 \mathrm{H}_{2}=10.0,6.0,3.0$ or $2.0 \mathrm{~mm}$, corresponding to contraction ratios of $2.4,4,8$ and 12 , respectively. We define the contraction ratio, $C R$, as the ratio between the upstream and the downstream side lengths of the square ducts, $C R=H_{1} / H_{2}$. The upstream and downstream sections are denoted by subscripts 1 and 2 , respectively. The flow rate is set by varying the height between the two

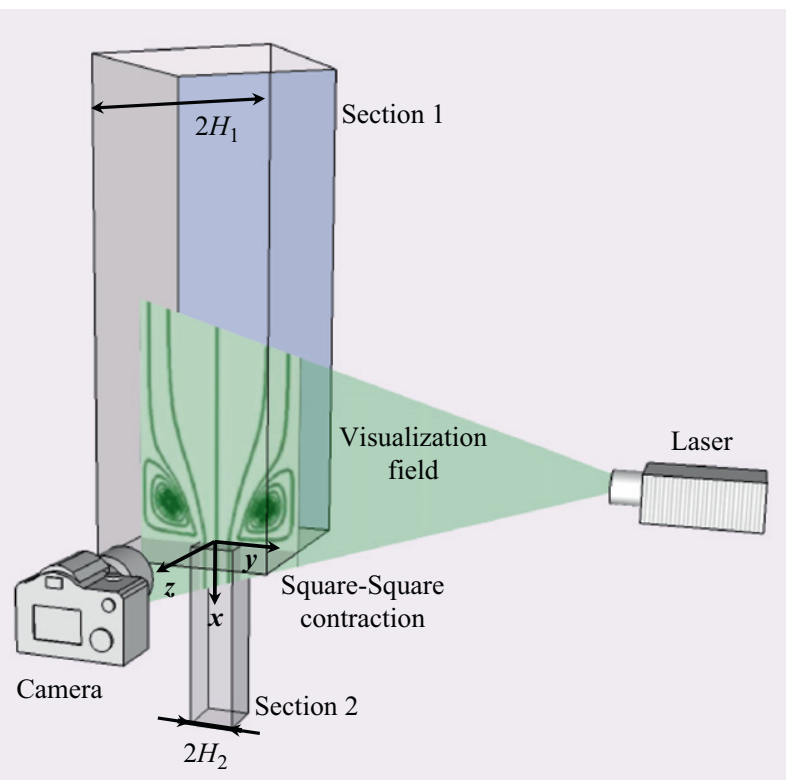

Fig. 1. Three-dimensional schematic representation of the experimental set-up used for flow visualization and particle image velocimetry measurements. 
free surfaces of the reservoirs or using a vacuum pump (KNF Loboport N811-KT.18) that applies vacuum to the outlet reservoir. For both cases, the selection of the diameter of the pipe that connects the duct to this outlet reservoir is also important to set the desired flow rate. To avoid degradation of the viscoelastic fluids used, flow regulating valves were not used in the experimental set-up. Further details on the experimental set-up can be found in Sousa et al. (2009).

The flow patterns were visualized using long time exposure streak line photography, while detailed velocity field measurements were performed using PIV. For this purpose, the working fluids were seeded with a low concentration of $10 \mu \mathrm{m}$ PVC tracer particles. In order to illuminate the plane of the flow under study, we used either a $3 \mathrm{~mW}$ $532 \mathrm{~nm}$ laser diode (Imatronic, model LLM115) or a $5 \mathrm{~mW} 635 \mathrm{~nm}$ laser diode (Vector, model 5200-20), both fitted with a cylindrical lens to create a light sheet. The streak line images were captured using a Canon EOS 30D digital camera equipped with a macro lens (Canon $\mathrm{EF} 100 \mathrm{~mm}, \mathrm{f} / 2.8$ ) and the exposure time was varied from about 1 to $10^{3} \mathrm{~s}$, depending on the flow rates.

For the PIV measurements, and for the flow visualizations at high flow rates, a double pulsed Nd:YAG laser with a maximum energy of $50 \mathrm{~mJ}$ (Solo PIV III, New Wave Research) was used to illuminate the channel and a digital CCD camera (Flow Sense 2M from Dantec Dynamics coupled with a Nikon AF Micro $60 \mathrm{~mm}$ lens), which was placed perpendicularly to the light sheet, was used to record the images. Time interval between pulses was varied in the range of $10^{-4} \leq \Delta t / s \leq 1$ depending on the flow rates. The images were acquired and postprocessed by FlowManager v4.60 software (Dantec Dynamics) using adaptive correlation on interrogation windows ranging between 64 by 64 pixels to 16 by 16 pixels, with $50 \%$ overlap.

\section{Fluid characterization}

A Newtonian fluid and a viscoelastic fluid with a shear-thinning rheological behavior were used in this study. The Newtonian fluid, used for comparison purposes, is an aqueous solution of glycerol $(85 \% \mathrm{w} / \mathrm{w})$ and the non-Newtonian fluid is a solution composed of $40.0 \%(\mathrm{w} / \mathrm{w})$ of glycerol, $59.9 \%(\mathrm{w} / \mathrm{w})$ of water and PAA at a weight concentration of $600 \mathrm{ppm}$. In order to minimize bacteriological growth in the fluids, and consequently to prevent its degradation, a biocide (Kathon LXE, Rohm and Haas) at a weight concentration of $25 \mathrm{ppm}$ was added to all solutions. The density $(\rho)$ of the Newtonian and viscoelastic fluids, measured at $293.2 \mathrm{~K}$ using a hydrometer (ranges $1200-1300$ and $1100-1200 \mathrm{~kg} / \mathrm{m}^{3}$; readability of $0.001 \mathrm{~kg} / \mathrm{m}^{3}$ ), were 1221 and $1156 \mathrm{~kg} \mathrm{~m}^{-3}$, respectively.

The fluids were characterized rheologically using a shear rheometer (Physica MCR301, Anton Paar) under shear rate control. The flow curves were measured at different temperatures $(T)$ ranging from 283.2 to $303.2 \mathrm{~K}$. The effect of the temperature on the shear viscosity can be described using an Arrhenius equation for the shift factor, $a_{T}$

$\ln \left(a_{T}\right)=\frac{\Delta H}{R}\left(\frac{1}{T}-\frac{1}{T_{0}}\right)$

where $\Delta H$ is the activation energy for flow, $R$ the universal gas constant and $T_{0}$ the reference temperature. We selected $T_{0}=293.2 \mathrm{~K}$, the average temperature at which the experiments were carried out, since the temperature of the measurements varied from 293.0 to 294.7 K. The shift factor is generally defined as (Dealy and Plazek, 2009)

$a_{T}=\frac{\eta(T)}{\eta\left(T_{0}\right)} \frac{T_{0}}{T} \frac{\rho_{0}}{\rho}$

in which $\eta\left(T_{0}\right)$ is the shear viscosity at the reference temperature, $\eta(T)$ is the shear viscosity at a given temperature $T$ and $\rho_{0}$ and $\rho$ are the fluid densities at the reference temperature, and at temperature $T$,

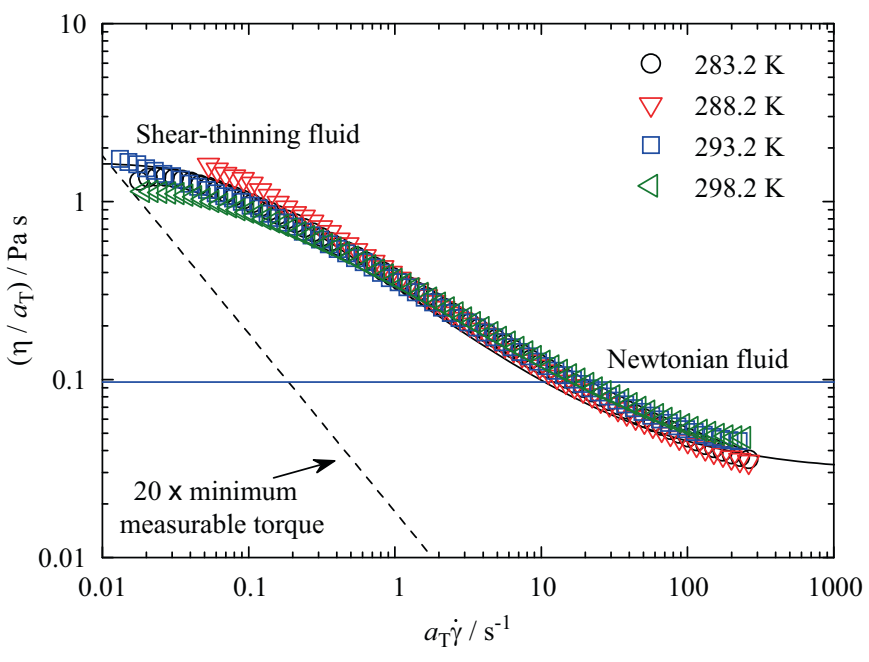

Fig. 2. Steady shear viscosity of the Newtonian and the shear-thinning fluid. For the shear-thinning fluid, the symbols represent the experimental data measured at different temperatures and the thick solid line the PTT model predictions. For the Newtonian fluid, the thin solid line represents the shear viscosity of the fluid at $293.2 \mathrm{~K}$. The minimum measurable shear viscosity based on 20 times the minimum resolvable torque is represented by the dashed line.

respectively. Nevertheless, in the range of measurements performed, the temperature variation is small and consequently the fluid density does not change significantly. As a result, the shift factor can be described as

$a_{T}=\frac{\eta(T)}{\eta\left(T_{0}\right)}$

For the Newtonian fluid, $\Delta H / R=5580 \mathrm{~K}$ and $\eta_{0}=0.0982 \mathrm{~Pa} \mathrm{~s}$ at the reference temperature. For the viscoelastic fluid, the flow curve was measured also at various temperatures, ranging from 283.2 to $298.2 \mathrm{~K}$, using a cone-plate with a $50 \mathrm{~mm}$ in diameter and $2^{\circ}$ angle. Subsequently, the time-temperature superposition principle (Dealy and Plazek, 2009) was used to obtain the master curve at the reference temperature $\left(T_{0}=293.2 \mathrm{~K}\right)$. In this case, a ratio of $\Delta H / R=2703 \mathrm{~K}$ was obtained. Fig. 2 shows the resulting steady shear viscosity master curve as well as the corresponding fit of a simplified Phan-ThienTanner model (Phan-Thien and Tanner, 1977) with a solvent viscosity contribution. A detailed description of this viscoelastic model is given in Section 4.

The parameters of the PTT model used in the fit presented in Fig. 2 are: extensibility parameter, $\varepsilon=0.06$; polymer shear viscosity coefficient, $\eta_{P}=1.62 \mathrm{~Pa} \mathrm{~s}$; solvent shear viscosity, $\eta_{S}=0.03 \mathrm{~Pa}$; relaxation time, $\lambda=32 \mathrm{~s}$.

\section{Numerical method and computational meshes}

In order to describe the isothermal and laminar flow of Newtonian and non-Newtonian incompressible fluids through squaresquare contractions, the following equations need to be solved: the conservation of mass and momentum,

$\nabla \cdot \mathbf{u}=0$

$\rho\left[\frac{\partial \mathbf{u}}{\partial t}+\nabla \cdot \mathbf{u u}\right]=-\nabla p+\eta_{S} \nabla^{2} \mathbf{u}+\nabla \cdot \tau$

and an adequate constitutive equation for the polymeric contribution $(\tau)$ to the extra stress tensor. In these equations, $\mathbf{u}$ is the velocity vector, $t$ the time, $p$ the pressure and $\eta_{S}$ the Newtonian solvent viscosity. The solvent contribution to the extra stress tensor, $\tau_{S}=\eta_{S}$ $\left(\nabla \mathbf{u}+\nabla \mathbf{u}^{\mathrm{T}}\right)$, is added explicitly in the momentum equation since this diffusive term $\left(\eta_{S} \nabla^{2} \mathbf{u}\right)$ promotes numerical stability. 
The rheological model used to fit the experimental shear data of the viscoelastic fluid was the simplified form of the Phan-ThienTanner model (cf. Section 3) and consequently, the polymeric contribution to the extra-stress tensor is given by (Phan-Thien and Tanner, 1977)

$f(\operatorname{Tr} \tau) \tau+\lambda\left[\frac{\partial \tau}{\partial t}+\nabla \cdot \mathbf{u} \tau\right]=\eta_{P}\left(\nabla \mathbf{u}+\nabla \mathbf{u}^{\mathrm{T}}\right)+\lambda\left(\tau \cdot \nabla \mathbf{u}+\nabla \mathbf{u}^{\mathrm{T}} \cdot \tau\right)$

The linear form of the PTT model was used, with the stress function given by (Phan-Thien and Tanner, 1977)

$f(\operatorname{Tr} \tau)=1+\frac{\lambda \varepsilon}{\eta_{P}} \operatorname{Tr}(\tau)$

where $\operatorname{Tr}(\tau)$ represents the trace of the extra-stress tensor. The simplified form of the Phan-Thien-Tanner model predicts a zero second-normal stress difference $\left(N_{2}\right)$ in fully developed shear flow, shear-thinning in steady shear flow and strain hardening in extensional flow, with the steady-state extensional viscosity being inversely proportional to the extensibility parameter, $\eta_{E} \propto 1 / \varepsilon$, for low values of $\varepsilon$. The fully developed flow of viscoelastic fluids with non-zero $\mathrm{N}_{2}$ shows a secondary flow in the transverse directions for non-circular ducts, as discussed by Yue et al. (2008). In the experiments we did not observe secondary flow under fully developed flow conditions, an indirect indication that the viscoelastic fluid has a negligible $N_{2}$, and the adequacy of using the simplified form of the Phan-Thien-Tanner model.

An alternative formulation for the constitutive equation uses the conformation tensor, $\mathbf{A}$, which can be related to the extra-stress tensor as

$\tau=\frac{\eta_{P}}{\lambda}(\mathbf{A}-\mathbf{I})$

where I represents the unitary tensor. For the PTT model the evolution equation for $\mathbf{A}$ is

$\lambda\left(\frac{\mathrm{DA}}{\mathrm{D} t}-\mathbf{A} \cdot \nabla \mathbf{u}-\nabla \mathbf{u}^{\mathrm{T}} \cdot \mathbf{A}\right)=-Y(\operatorname{Tr} \mathbf{A})(\mathbf{A}-\mathbf{I})$

with $Y(\operatorname{Tr} \mathbf{A})=1+\varepsilon(\operatorname{Tr} \mathbf{A}-3)$.

Eq. (9) preserves the positive definiteness of $\mathbf{A}$, and using this important property allows the use of the logarithm of the conformation tensor, $\boldsymbol{\Theta}=\log \mathbf{A}$, which can alleviate the numerical difficulties related with exponential growth of stress profiles of highly elastic flows, as proposed by Fattal and Kupferman (2004). This transformation, known as the log-conformation formulation, improves the stability of the numerical method and alleviates the high-Weissenberg number problem (HWNP), making possible to achieve converged solutions at higher Deborah (or Weissenberg) numbers (e.g. Hulsen et al., 2005). Eq. (9) can be re-written in terms of the logarithm of the conformation tensor (Fattal and Kupferman, 2004)

$\lambda\left[\frac{\partial \Theta}{\partial t}+\mathbf{u} \cdot \nabla \boldsymbol{\Theta}-(\mathbf{R} \Theta-\mathbf{\Theta R})-2 \mathbf{E}\right]=Y\left[\operatorname{Tr}\left(\mathrm{e}^{\boldsymbol{\Theta}}\right)\right]\left(\mathrm{e}^{-\boldsymbol{\Theta}}-\mathbf{I}\right)$

where $\mathbf{R}$ and $\mathbf{E}$ are a pure rotational tensor and a traceless extensional tensor, respectively, which combine to form the velocity gradient tensor (Fattal and Kupferman, 2004). After solving Eq. (10) for $\Theta$, the conformation tensor can be recovered using the inverse transformation, $\boldsymbol{A}=\mathrm{e}^{\boldsymbol{\Theta}}$, and the extra-stress can be calculated using Eq. (8).

An implicit finite volume method (FVM) was used to solve numerically the set of Eqs. (4), (5) and (10). The governing equations are integrated in space over the control volumes of the mesh, and integrated in time over small time increments $(\delta t)$, leading to linearized algebraic equations to be solved for the logarithm of the conformation tensor components, $\Theta_{i j}$,

$a_{P}^{\Theta} \Theta_{i j, P}=\sum_{F=1}^{6} a_{F}^{\Theta} \Theta_{i j, F}+S_{\Theta_{i j}}$

and for the velocity components, $u_{i}$,

$a_{P} u_{i, P}=\sum_{F=1}^{6} a_{F} u_{i, F}+S_{u_{i}}$

In Eqs. (11) and (12) $a_{P}^{\Theta}$ and $a_{P}$ represent the central coefficients, and the coefficients of the neighbor cells $a_{F}$ account for advection and diffusion (no diffusion in $a_{F}^{\Theta}$ due to the inexistence of diffusion term in Eq. (10)). The summation is carried out over the six neighbor cells ( $F$ ) that have a face in common with cell $P$ under analysis. The source terms in Eqs. (11) and (12) include all the terms that are evaluated explicitly, based on previous time-step values. In summary, the numerical methodology consists of the following steps:

1. The conformation tensor $A_{i j}$ is calculated from the extra-stress components $\tau_{i j}$ (using Eq. (8)), and the log-conformation tensor $\Theta_{i j}$ is calculated from $A_{i j}$ (cf. Afonso et al., 2009 for details).

2. The evolution equation for $\Theta_{i j}$ (Eq. (10)) is solved implicitly to obtain the new time level values of $\Theta_{i j}$.

3. The conformation tensor is calculated from $\Theta_{i j}$ and the extrastress tensor at the new time level is computed using Eq. (8).

4. The momentum equation is solved for the three Cartesian components of the velocity vector, $u_{i}$.

5. The computed velocity field usually does not verify the continuity equation, and a correction of $u_{i}$ and pressure is done to simultaneously verify the continuity and momentum equations, following the SIMPLEC methodology (van Doormal and Raithby, 1984). 6. Steps 1-5 are repeated until convergence is achieved. a

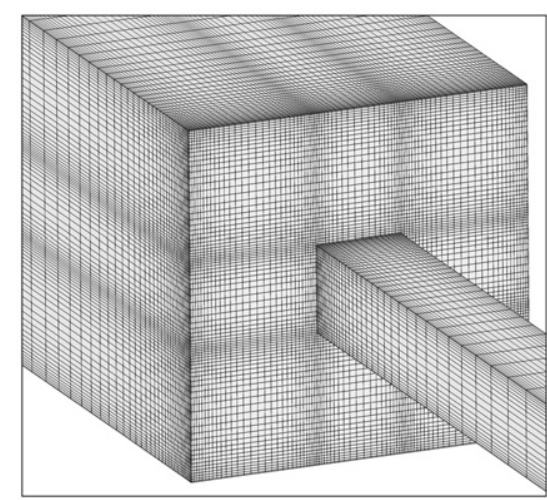

b

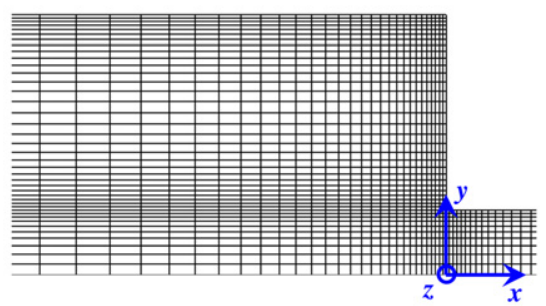

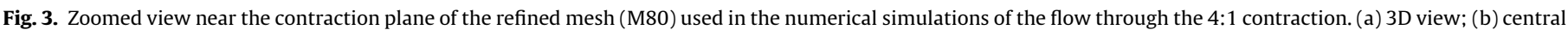
plane, $z=0$ (only half of the plane is shown). 
Regarding the accuracy of the calculations, an implicit firstorder Euler scheme is used in the discretization of the time derivative, second-order central differences are used to discretize the diffusive terms and the CUBISTA high-resolution scheme (Alves et al., 2003a) is used in the discretization of the advective terms in the momentum and constitutive equations.

The simulations were performed on three-dimensional meshes covering the whole wall-to-wall geometries, i.e. no symmetry at the central and/or diagonal planes was enforced. The meshes consist of orthogonal blocks which are composed of non-uniform cells. A collocated mesh arrangement is used, i.e. all variables are calculated at the center of the cells, avoiding that the stresses (or the logarithm of the conformation tensor) are calculated exactly on the re-entrant corners of the contraction, where the stresses become unbounded (singular point), as demonstrated theoretically by Evans and Sibley (2008) and numerically by Alves et al. (2003b) for a planar contraction.

The inlets and outlets were positioned far from the contraction plane so that fully developed flow conditions were attained. No-slip boundary conditions were imposed at the solid walls and a uniform velocity profile and null stress components were imposed at the inlet boundary. The outflow boundary condition involves vanishing stream wise gradients of velocity and stress components and linear extrapolation of pressure from the two upstream computational cells.

Fig. 3 shows a zoomed view, near the contraction plane, of the refined mesh used in the simulation of the flow through the $4: 1$ square-square contraction. To assess numerical accuracy, three different computational meshes were used for $C R=2.4$ and 4 (meshes M40, M64 and M80) and two meshes were used for $C R=8$ (meshes M40 and M64) and $C R=12$ (meshes M48 and M60).

\section{Table 1}

Characteristics of the computational meshes used.

\begin{tabular}{lllll}
\hline$C R$ & Mesh & NC & $\Delta x_{\min } / 2 H_{1}$ & $\begin{array}{l}\Delta y_{\min } / 2 H_{1}= \\
\Delta z_{\min } / 2 H_{1}\end{array}$ \\
\hline \multirow{2}{*}{2.4} & M40 & 164000 & $2.08 \times 10^{-2}$ & $1.99 \times 10^{-2}$ \\
& M64 & 419840 & $1.30 \times 10^{-2}$ & $1.25 \times 10^{-2}$ \\
& M80 & 656000 & $1.03 \times 10^{-2}$ & $9.93 \times 10^{-3}$ \\
4 & M40 & 51000 & $1.31 \times 10^{-2}$ & $1.25 \times 10^{-2}$ \\
& M64 & 130560 & $8.20 \times 10^{-3}$ & $8.16 \times 10^{-3}$ \\
& M80 & 408000 & $6.26 \times 10^{-3}$ & $6.25 \times 10^{-3}$ \\
& M40 & 163200 & $7.50 \times 10^{-3}$ & $7.50 \times 10^{-3}$ \\
& M64 & 417792 & $4.69 \times 10^{-3}$ & $4.55 \times 10^{-3}$ \\
& M48 & 113664 & $1.07 \times 10^{-2}$ & $9.52 \times 10^{-3}$ \\
& M60 & 177600 & $8.61 \times 10^{-3}$ & $8.33 \times 10^{-3}$ \\
\hline
\end{tabular}

Details of the total number of cells (NC) as well as the cell smallest dimensions $\left(\Delta x_{\min } / 2 H_{1}, \Delta y_{\min } / 2 H_{1}\right.$ and $\left.\Delta z_{\min } / 2 H_{1}\right)$ for each mesh are described in Table 1 . The reference number identifying each mesh (e.g. M40) corresponds to the number of cells along each transverse direction in the upstream channel.

\section{Flow patterns and vortex length}

\subsection{Newtonian fluid}

A detailed discussion of the Newtonian fluid flow through square-square contractions with different contraction ratios was documented in a previous work (Sousa et al., 2009). Here, we focus only on the main results with the purpose of comparing the flow of the Newtonian and the shear-thinning fluids.

In order to characterize the Newtonian fluid flow we use the Reynolds number, here defined as $\operatorname{Re}=\rho U_{2}\left(2 \mathrm{H}_{2}\right) / \eta$, where $U_{2}$ is the average velocity in the downstream channel. In Fig. 4 we show the flow patterns obtained for the Newtonian fluid flow through the 8:1 squaresquare contraction for two Reynolds numbers ( $R e=2.13$ and 13.7). Moreover, in Fig. 4 we compare the experimental results with numerical predictions highlighting the variation of the vortex length with increasing flow inertia. The vortex length, $x_{R}$, is here defined as the distance from the separation point to the contraction plane, as shown in Fig. 4(a).

The Newtonian fluid flow behavior is similar for all contraction ratios studied ( $C R=2.4,4$ and 8 ). A corner vortex appears upstream of the contraction plane which is typical of flows through abrupt contractions (cf. Oliveira et al., 2007 for the axisymmetric arrangement and Alves et al., 2003b for the planar configuration) and agrees with the findings of Sirakov et al. (2005) and Alves et al. (2005) for 3D geometries. Furthermore, the size of the upstream vortex decreases when the Reynolds number is increased. The comparison between the experimental and numerical results demonstrates that there is an excellent agreement between both approaches.

\subsection{Shear-thinning viscoelastic fluid}

Besides the contraction ratio and the Reynolds number, an important dimensionless number that should be taken into account for the characterization of the viscoelastic fluid flow is the Deborah number, which is here defined as $D e=\lambda U_{2} / H_{2}$. Due to the shearthinning behavior of the viscoelastic fluid, the Reynolds number is now defined as $\operatorname{Re}=\rho U_{2}\left(2 \mathrm{H}_{2}\right) / \eta(\dot{\gamma})$, where the shear viscosity is a

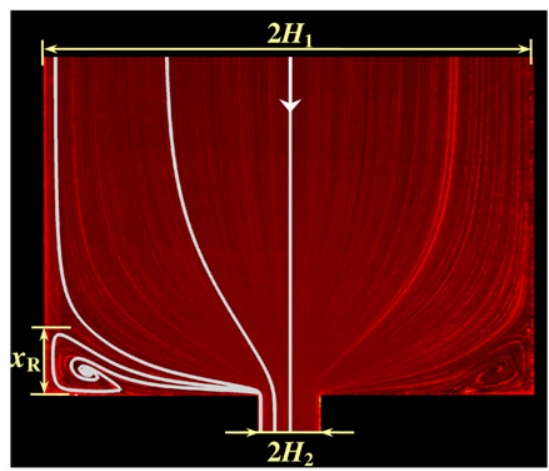

b

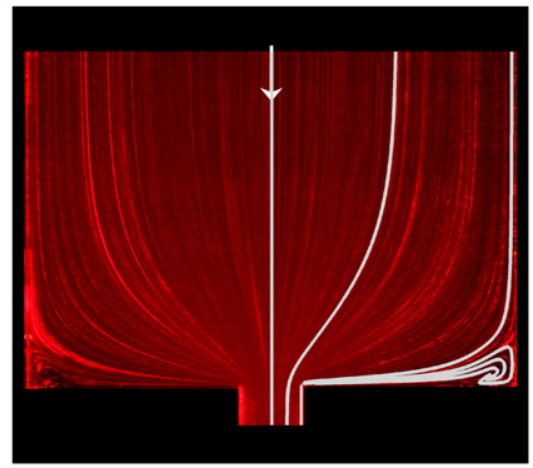

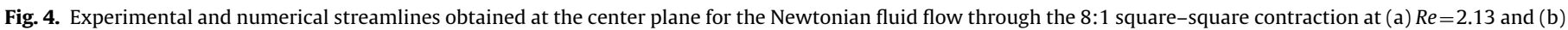
$R e=13.7$. 
calculated using the PTT model at the characteristic shear rate $\dot{\gamma}=U_{2} / H_{2}$.

Fig. 5 shows the flow patterns for a low and a high contraction ratio ( $C R=2.4$ and 12 ) for different Deborah numbers, which were varied by changing the flow rate. The Reynolds number also varies with the flow rate, but in all cases inertial effects are not important because $R e$ is small for the range of flow conditions studied with the viscoelastic fluid.

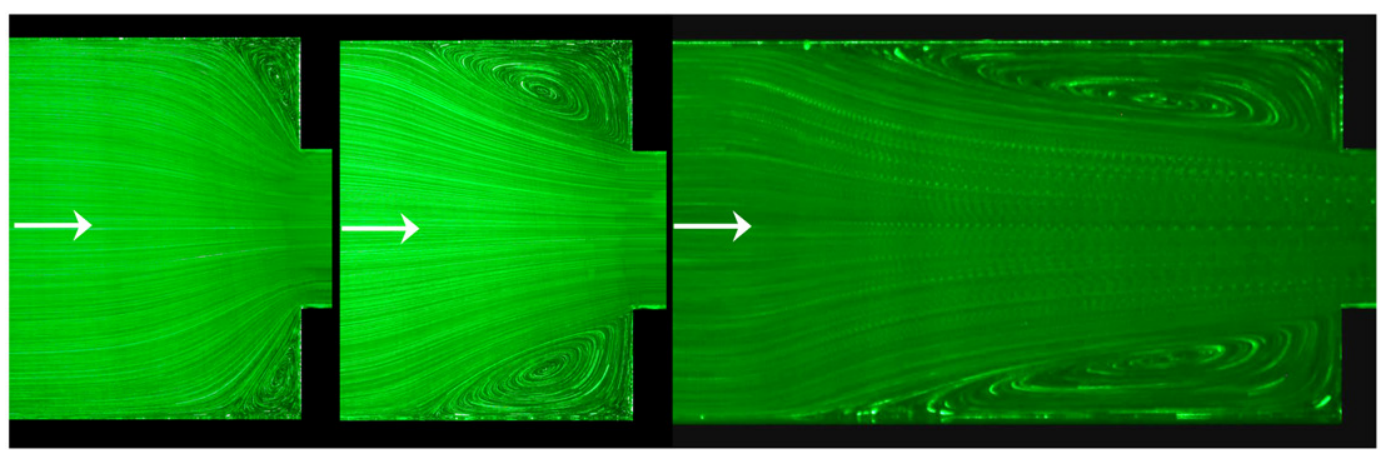
(a1) $R e=0.00136$ $D e=1.07$
(a2) $R e=0.0280$
$D e=9.63$
(a3) $R e=0.697$
$D e=77.3$

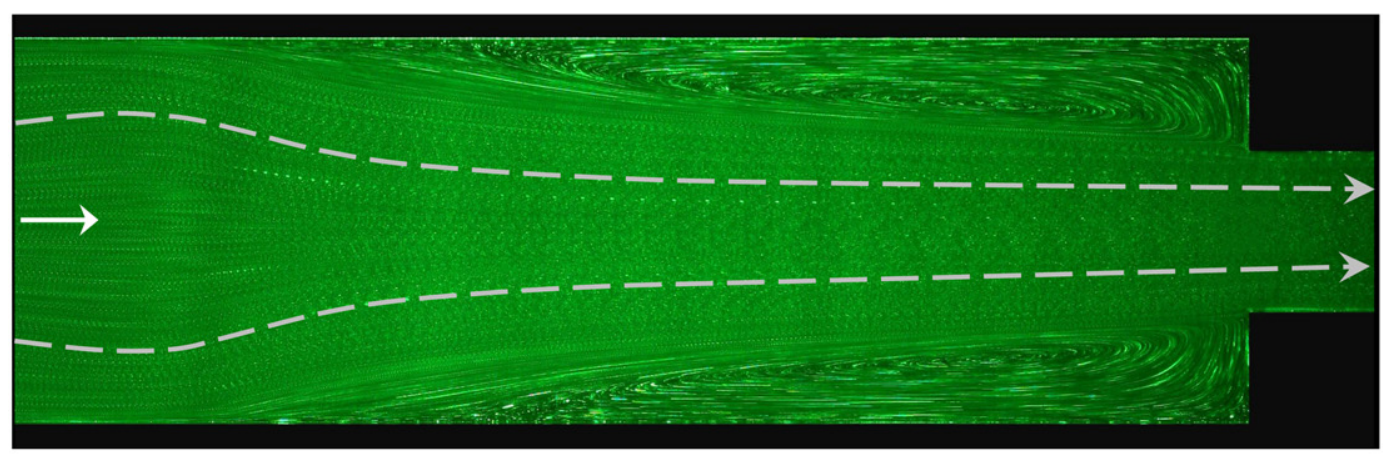

(a4) $R e=2.37 \quad D e=174$

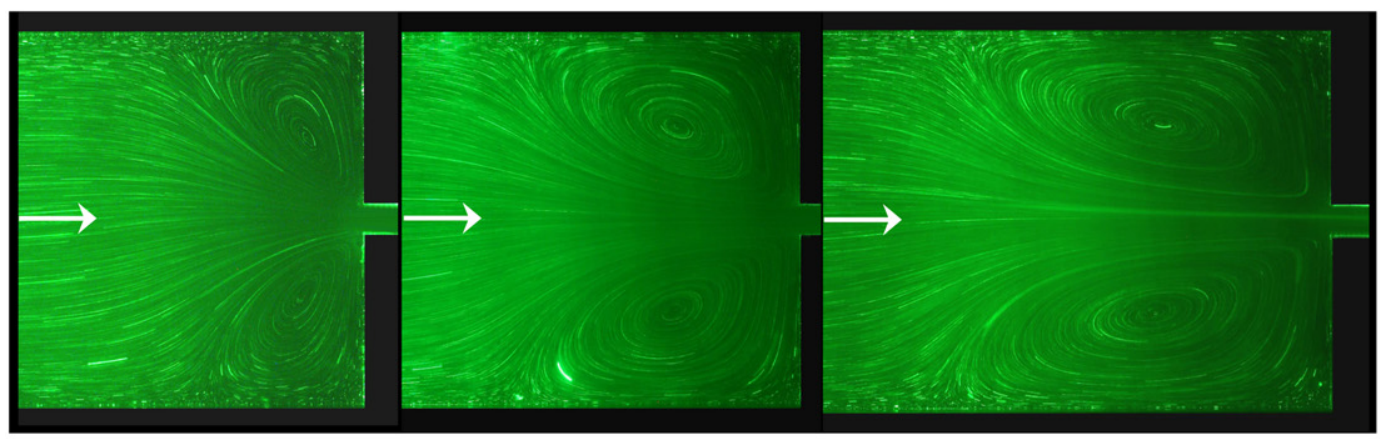
(b1) $R e=0.00120$
$D e=10.3$
(b2) $R e=0.0109$
(b3) $R e=0.0278$
$D e=42.8$
$\mathrm{De}=78.0$

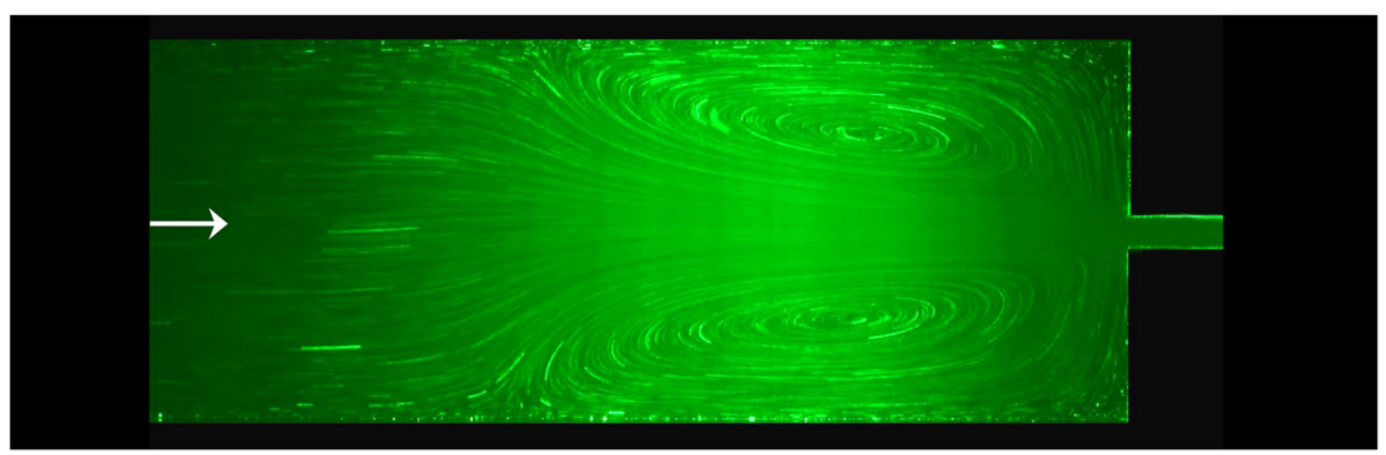

(b4) $R e=0.111 \quad D e=192$

Fig. 5. Flow patterns obtained with the viscoelastic fluid at the center plane for (a) $C R=2.4$ and (b) $C R=12$. The diverging streamlines which appear for $C R=2.4$ are highlighted by dashed lines (a4). 
For all $C R$ studied, at low flow rates (or $D e$ ), a Newtonian-like behavior is observed (cf. Fig. 5(a1)). Increasing the Deborah number, a strong vortex enhancement is observed (cf. Fig. 5(a2)-(a4) and (b1)-(b4)). For the lower contraction ratios investigated, $C R=2.4$ and 4 , this vortex enhancement is accompanied by the onset of diverging streamlines upstream of the contraction plane, which appear at $D e>10$ for $C R=2.4$ (cf. Fig. 5(a4)) and at $D e>20$ for $C R=4$. Divergent streamlines are often observed in viscoelastic contraction flows (Cable and Boger, 1978a, b, 1979; Evans and Walters, 1989, McKinley et al., 2007) and are usually enhanced at lower contraction ratios (Alves and Poole, 2007), as confirmed here. In fact, in the present work divergent flow was not observed for the higher contraction ratios, $C R=8$ and 12 . Furthermore, the small values of the Reynolds numbers used in this work suggest that inertia is not a fundamental condition for the appearance of diverging flow, in agreement with the findings of Alves and Poole (2007).

The vortex length at the center plane of the square channel, $x_{R}$, was measured for all flow rates and contraction ratios studied, using the streak line images captured experimentally as well the velocity field predicted from numerical simulations. Fig. 6 shows the variation of the dimensionless vortex length at the center plane $\left(x_{R} / 2 H_{1}\right)$ as a function of the Deborah number for all the contraction ratios investigated. The recirculations that appear upstream of the contraction increase in size with an increase in the flow rate (or Deborah number), in contrast with Newtonian fluid flow, in which the vortex length decreases slightly with the flow rate, as shown in Section 5.1. For all $C R$, but particularly for the lower contraction ratios studied in this work $(C R=2.4$ and 4$)$, the vortex length increases considerably, exceeding three times the width of the upstream channel. The vortex enhancement observed in the flow of a shear-thinning fluid through contractions was previously documented in the works of Alves et al. (2008) for a 4:1 squaresquare contraction, by Evans and Walters (1986) for planar and square-square abrupt contractions and by Cable and Boger (1978a) for axisymmetric contractions. For Boger fluids vortex enhancement is usually observed in axisymmetric and in square-square contractions (Evans and Walters, 1986; Alves et al., 2005), while for planar contractions vortex activity is suppressed or not significant (Walters, 1985; Evans and Walters, 1986). This difference in flow behavior can be related with the higher Hencky strains experienced in square-square and axisymmetric contractions $\left(\varepsilon_{H}=2 \ln C R\right)$, in comparison to the planar arrangement $\left(\varepsilon_{H}=\ln C R\right)$.

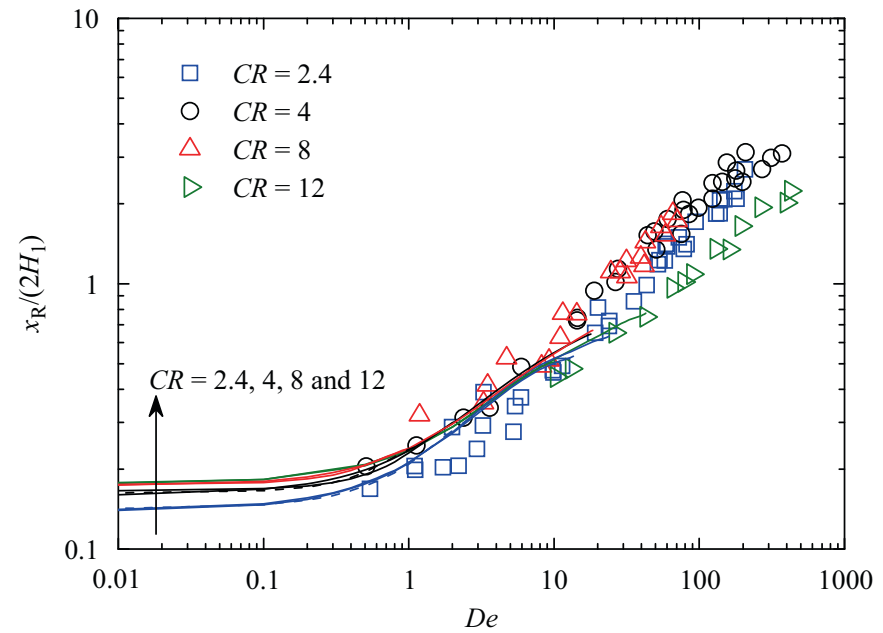

Fig. 6. Dimensionless vortex length at the center plane as a function of the Deborah number for all contraction ratios studied. The symbols represent the experimental data, the thick solid lines represent the numerical predictions using mesh M40 (M48 for $C R=12$ ), the thin solid lines the predictions using mesh M64 (M60 for $C R=12$ ) and the dashed lines the predictions using mesh M80.
The numerical results presented in Fig. 6 for $C R=2.4$ and 4 were obtained using the three meshes described in Section 4 (M40, M64 and M80). For the range of De where steady solutions were obtained in the numerical simulations, the vortex lengths predicted numerically are in good agreement with the experimental values. Moreover, the differences between meshes M64 and M80 are small (relative deviation of about $1 \%$ ). For this reason and since numerical simulation of the viscoelastic fluid flow using the more refined mesh is time-consuming, for the higher contraction ratios, $C R=8$ and 12 , we only show the numerical results obtained using mesh M40 or M48 and M64 or M60, respectively. Average computational times for $C R=2.4$ were 15 hours using mesh M40, 6 days using mesh M64 and 4 weeks in mesh M80, in all cases using a desktop computer with an Intel Core2 Quad Processor Q9400 running at 2.66 GHz, and with $4 \mathrm{~GB}$ of RAM. For the other contraction ratios similar CPU times were necessary to achieve convergence in each mesh. The maximum Deborah number $\left(D e_{\max }\right)$ achieved in the numerical simulations varied with $C R$ and mesh used. In mesh M40De $e_{\max }$ varied from 24 to 43 for $C R=2.4$ and 12, respectively, while for mesh M64 De $e_{\max }$ varied from 14 to 10 for $C R=2.4$ and 12 , respectively. In mesh M80 given the large CPU times involved, we restricted the simulations to a few De cases, in order to assess numerical accuracy and convergence with mesh refinement. The general trend observed, with $D e_{\max }$ decreasing with mesh refinement, has been reported in most of the numerical studies of viscoelastic fluid flow in contractions (Owens and Phillips 2002) and is related with the large gradients of the stress (or conformation) field observed near the walls, and particularly in the sharp (re-entrant) corners where the stress (and conformation) field is unbounded (singular point).

In the numerical calculations we are only able to probe a range of Deborah numbers much smaller than in the experiments, as a consequence of the HWNP and the limitations of the PTT model. Nevertheless, we are able to predict numerically the observed vortex enhancement as De increases with good accuracy. To further attest this, in Fig. 7 we show the numerical streamlines for the shear-thinning fluid flow through the 2.4:1 square-square contraction and a comparison with the flow visualization at high $D e$ to
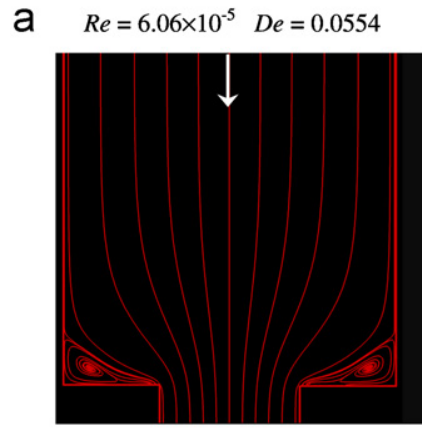

C

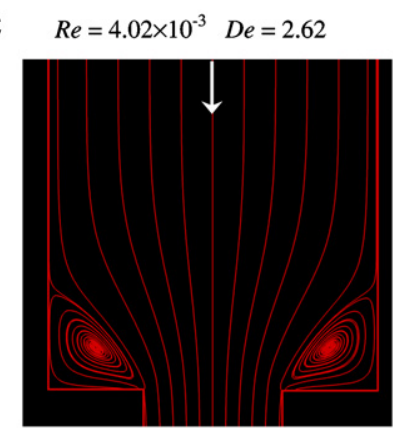

\section{b}

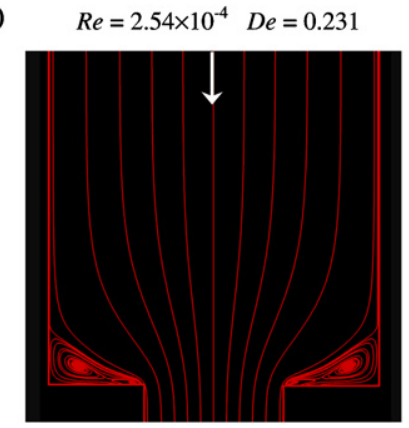

d

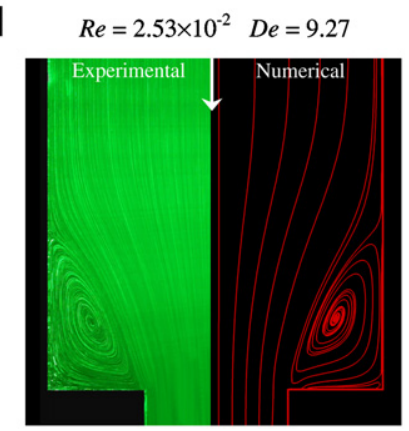

Fig. 7. Viscoelastic fluid flow patterns predicted numerically for $C R=2.4$ (mesh M64) at the center plane at different flow conditions and comparison with experimental results $(\mathrm{d})$. 
illustrate the good agreement between experiments and numerical predictions.

As explained previously, at low Deborah numbers the flow is Newtonian-like (compare Figs. 7(a) and 5(a1) for the viscoelastic fluid to Fig. 4(a) corresponding to the Newtonian fluid) and the upstream vortices are slightly concave-shaped. At $D e=0.231$, the numerical results show the presence of small lip vortices near the re-entrant corners which were not observed in the experimental results because visualizations were restricted to $D e>0.5$ due to experimental difficulties of operation at such low flow rates.

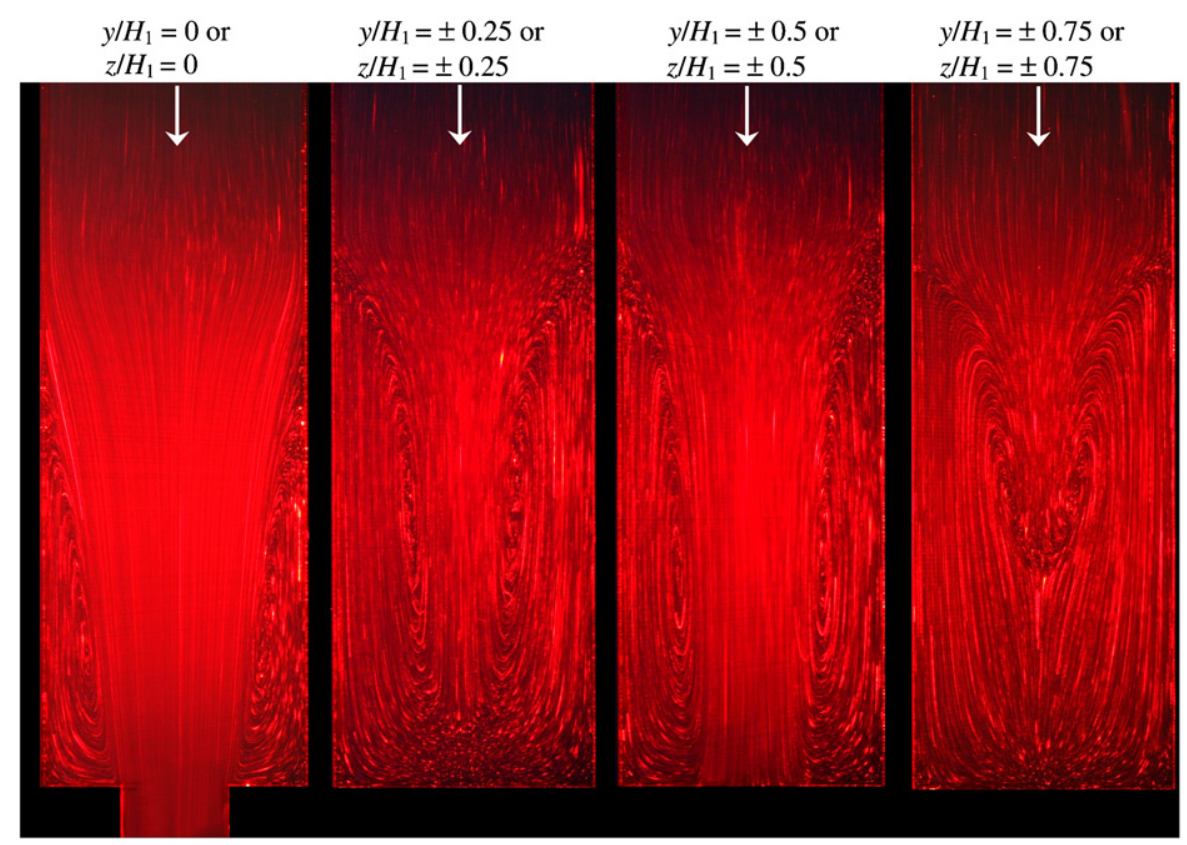

Fig. 8. Projected streamlines obtained for the viscoelastic fluid at different parallel planes of the $2.4: 1$ square-square contraction for $R e=0.930$ and $D e=92.0$.

a $y / H_{2}=0$ or $z / H_{1}=0$

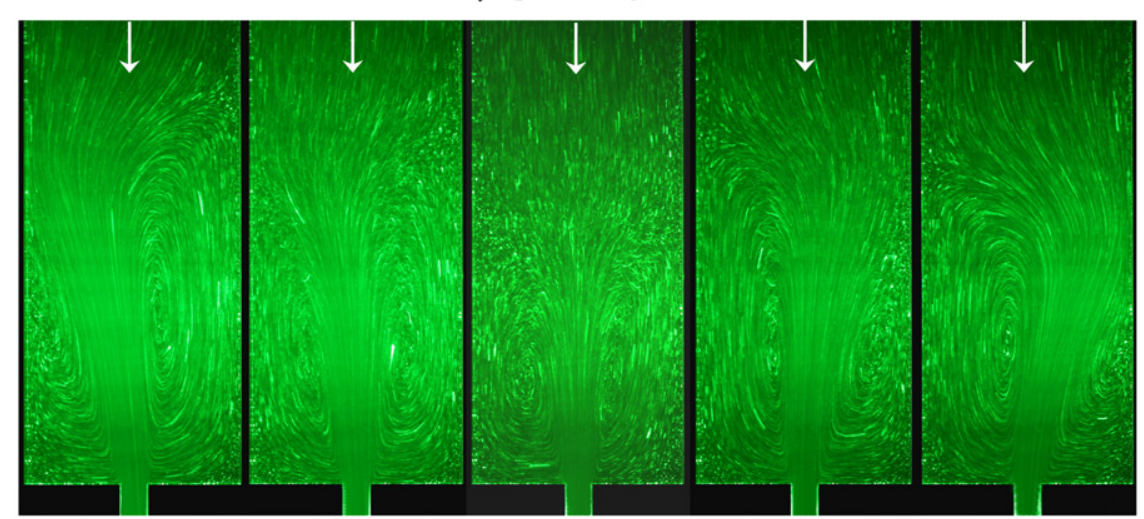

b

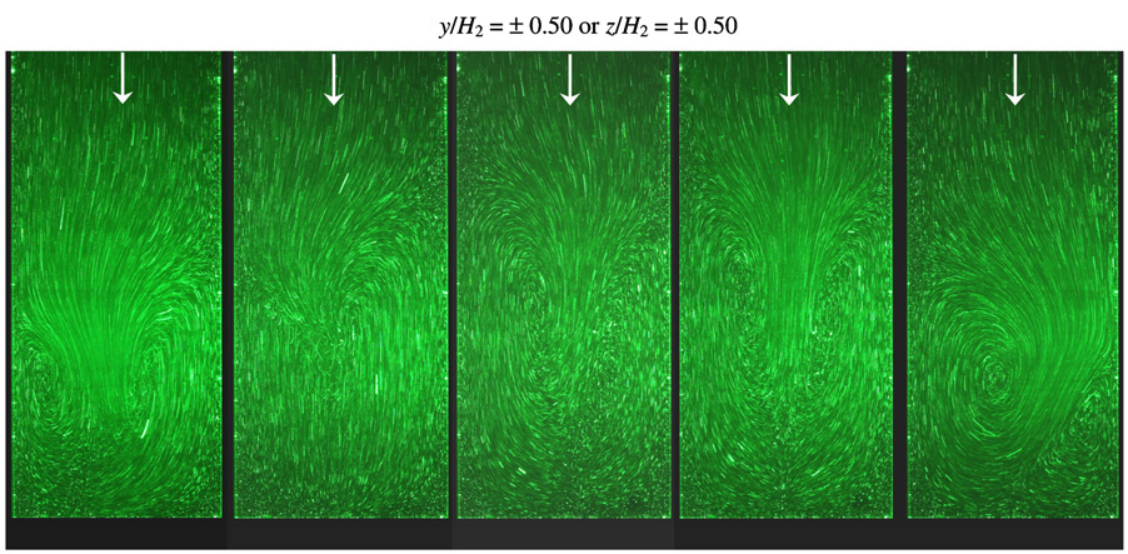

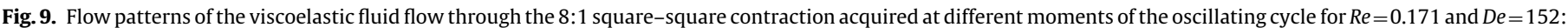
(a) center plane; (b) middle plane between the wall and the center plane. 
Increasing $D e$, the recirculations increase in size and become convex in shape (cf. Fig. 7(b) and (c)) in contrast with those obtained for Newtonian fluid flow. At a certain value of the Deborah number, there is a reversal of the flow direction within the 3D recirculation which is predicted numerically as can be seen in Fig. 7(c) and (d) where the fluid is clearly entering the recirculation in the middle plane, in contrast with the behavior of low De or Newtonian fluid flows (cf. Fig. 4), where the fluid exits the recirculation at the middle plane vortex.

For the lower $C R$, within the experimental range of flow rates measured, it is possible to establish that the inversion of the flow direction occurs at $D e \approx 3$ and 2 for $C R=2.4$ and 4 , respectively. For higher $C R$ we are not able to determine experimentally the value of De at which flow reversal takes place due to the absence of experimental data at those flow conditions. From numerical results we predict that this phenomenon occurs at $D e \approx 1.5$ for all $C R$. The same phenomenon was reported in the works of Alves et al. (2008) and of Sousa et al. (2009) for the flow through square-square contractions of a PAA-based shear-thinning and a Boger fluid, respectively. Additionally, flow reversal was also observed in square-square expansions (Sousa et al., submitted for publication) although in this case it only happened for Newtonian fluids, when inertial effects become important. The reported vortex enhancement due to elasticity in contraction flows, and due to inertia in expansion flows, seems to be linked with the flow reversal phenomenon.

Fig. 8 shows the projected streamlines at different parallel planes of the square channel, in order to illustrate the trajectory of the fluid within the recirculation for high De flows in the 2.4:1 squaresquare contraction. It is interesting to note that the recirculation structure is complex and that the eye of the recirculation moves upstream as the wall plane is approached.

When the flow rate (or $D e$ ) is increased further, an elastic instability occurs and the flow becomes time-dependent, with the size of the recirculations varying in time in a complex way, which eventually becomes chaotic at larger flow rates. In Fig. 9 we show a sequence of instantaneous pathlines visualized using the shear-thinning fluid for a Deborah number at which the flow is unstable, clearly showing that the vortices vary in size and shape with time. To further attest to the time dependency and the three-dimensional structure of the flow we also show in Fig. 9(b) instantaneous projections of pathlines at a plane located at middle-distance between the center plane and the wall of the square channel $\left(y / H_{2}= \pm 0.50\right.$ or $\left.z / H_{2}= \pm 0.50\right)$. As can be seen, the flow becomes asymmetric oscillating periodically, i.e. during a cycle of oscillation, one region of the vortex size decreases while the other part is increasing. From Fig. 9(b), it is possible to conclude that the entire 3D vortical structure is moving. The normalized frequency of oscillation, $\lambda f$, is plotted in Fig. 10 as a function of the ratio $D e / C R$, together with results reported in a previous work (Sousa et al., 2009) for a Boger fluid flowing through square-square contractions with $C R=4,8$ and 12 . A general trend can be identified with $\lambda f$ varying approximately linearly with $\log (\mathrm{De} / \mathrm{CR})$.

\subsection{Flow pattern map}

The different flow phenomena identified for the viscoelastic fluid flow through square-square contractions can be summarized in a $C R-D e$ map, as shown in Fig. 11.

Each flow regime is bounded between an upper and lower limit that are marked as dashed lines in Fig. 11. The distinct flow regimes observed with the shear-thinning fluid were categorized as:

- Newtonian-like flow for conditions of negligible elastic effects, in which the upstream vortices increase in size only slightly as the Deborah number increases.

- Vortex enhancement, in which the vortex increases significantly due to elastic effects.

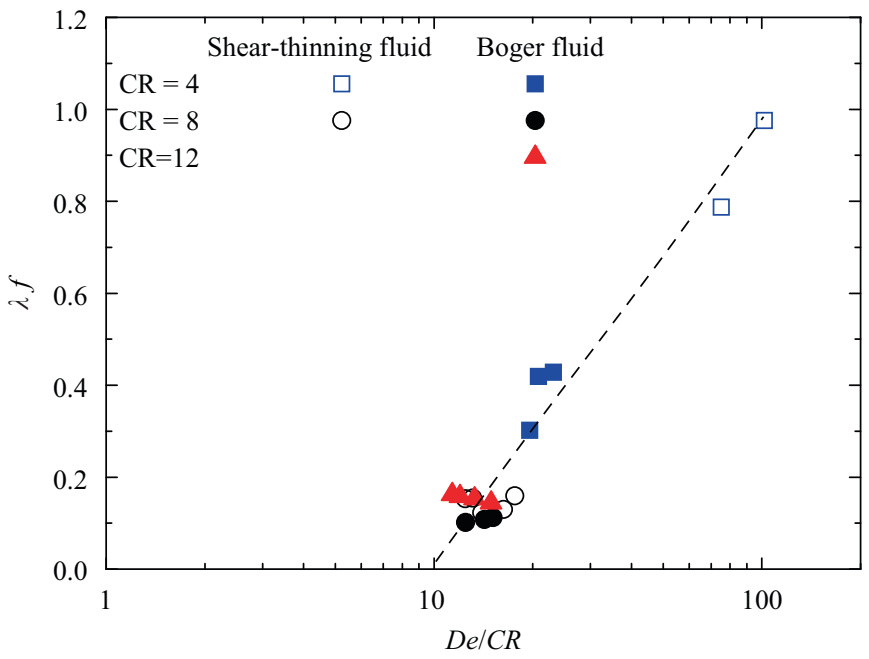

Fig. 10. Normalized frequency of oscillation as a function of $D e / C R$ for $C R=4,8$ and 12. The open symbols represent experimental data for the shear-thinning fluid and the filled symbols represent experimental data obtained by Sousa et al. (2009) for the Boger fluid flow through similar square-square contractions. The dashed line is a guide to the eye.

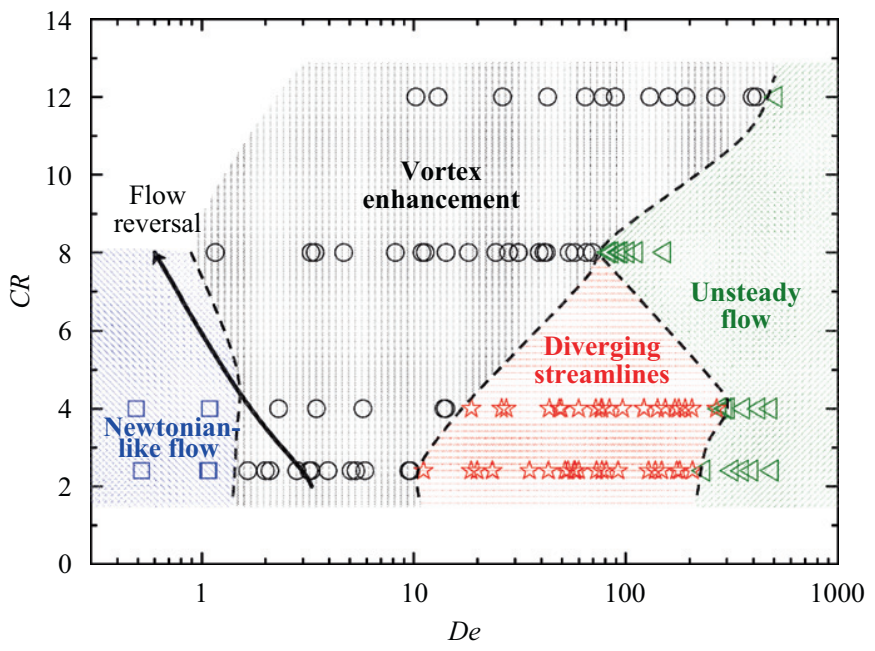

Fig. 11. Summary of the flow patterns identified in the $C R-D e$ parameter space for the shear-thinning viscoelastic fluid. The symbols represent experimental results and are identified as follows: squares: quasi-Newtonian flow; circles: elasticity driven vortex enhancement; stars: diverging streamlines upstream of contraction; triangles: unsteady flow.

- Diverging streamlines, which occurs for the lower contraction ratios ( $C R=2.4$ and 4 ) when the Deborah number (and consequently the elastic effects) is further increased. We note that this flow regime occurs simultaneously with vortex enhancement.

- Unsteady flow that occurs at higher Deborah numbers, in which the entire flow field oscillates periodically.

\section{Velocity field}

In order to highlight the effect of elasticity on the velocity field, in Fig. 12 we show dimensionless axial velocity profiles along the centerline $(y=z=0)$ for three contraction ratios at different Deborah numbers. In addition, the numerical prediction of the axial velocity profile for a Newtonian fluid flow under creeping flow conditions is also presented in Fig. 12 for comparison purposes, since this is the limiting case that would be observed with the viscoelastic fluid at very low flow rates. For the range of high Deborah numbers at which the 
a

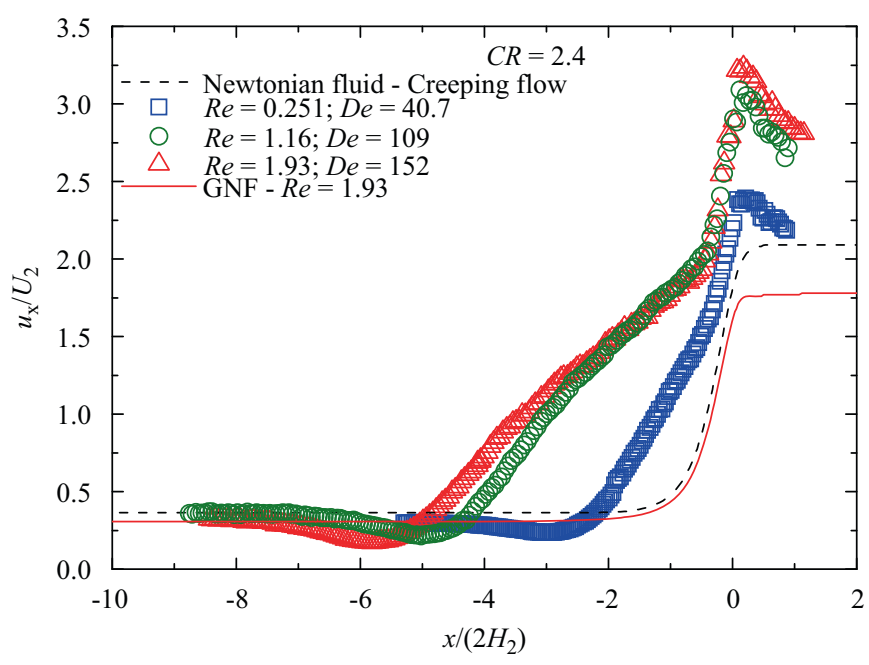

b

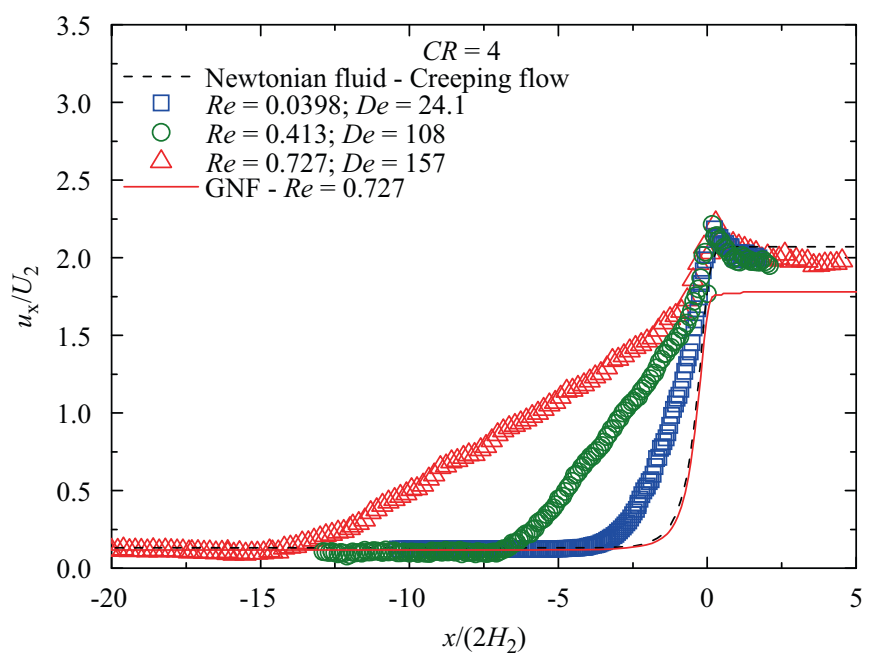

C

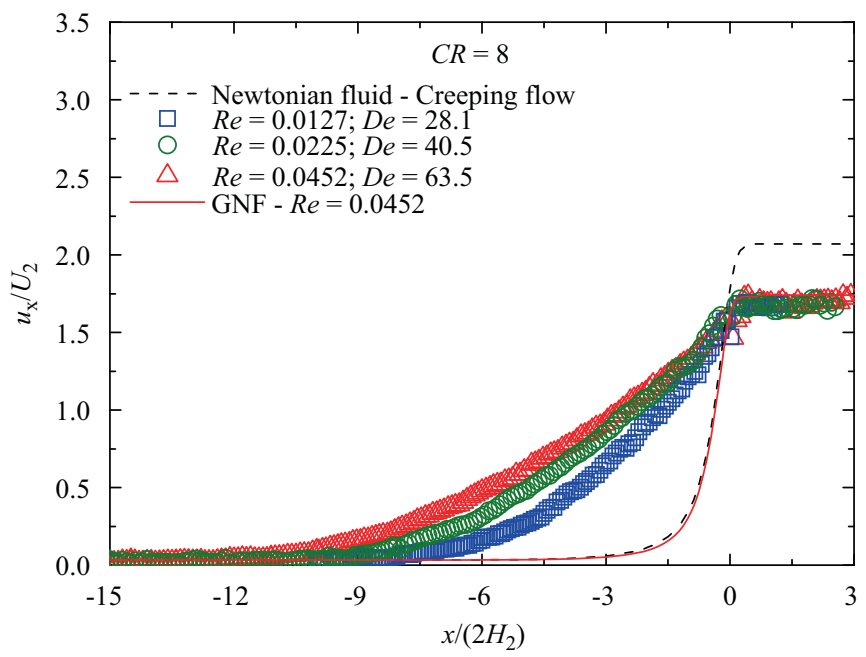

Fig. 12. Axial velocity profiles at the channel centerline for (a) $C R=2.4$, (b) $C R=4$ and (c) $C R=8$. The symbols represent the experimental data obtained with the viscoelastic fluid, the dashed line represents the numerical predictions for a Newtonian fluid under creeping flow conditions and the solid line represents the numerical predictions for the flow of a generalized Newtonian fluid.

experimental velocity profiles were measured using PIV, we are unable to obtain the velocity field numerically using the PTT model due to the high Weissenberg number problem. However, we have performed numerical calculations using a generalized Newtonian fluid (GNF), which does not account for elasticity, but presents a rheological behavior in steady shear flow similar to that of the viscoelastic fluid used in the experiments. For this purpose, a Carreau-Yasuda model (Bird et al. 1987) was used to fit the shear viscosity data of the GNF model

$\eta=\eta_{S}+\frac{\eta_{0}-\eta_{S}}{\left[1+(\Lambda \dot{\gamma})^{a}\right]^{(1-n) / a}}$

where the fitted parameters are $\eta_{0}=1.65 \mathrm{~Pa} \mathrm{~s}, \eta_{s}=0.03 \mathrm{~Pa} \mathrm{~s}, \Lambda=10 \mathrm{~s}$, $n=0.36$ and $a=0.9$.

For the inertialess Newtonian fluid flow the behavior is similar for all CR: upstream of the contraction the fluid experiences a constant axial velocity along the centerline, up to a location near the contraction plane, when the fluid accelerates and enters the downstream channel eventually reaching the conditions of developed flow, with $u / U_{2}=2.096$ at the centerline of a square channel (Sousa et al., 2009). For the viscoelastic fluid flow, the influence of elasticity leads to a different, and more complex, variation of the axial velocity along the centerline. At a location far upstream from the contraction plane, the value of the dimensionless axial velocity is similar to that found for the Newtonian fluid. However, for all contraction ratios studied the location at which the fluid starts to accelerate, due to entry effects, shifts significantly upstream with an increase with the Deborah number. For the lower contraction ratios $(C R=2.4$ and 4$)$ the presence of diverging streamlines leads to a velocity undershoot immediately before the fluid starts to accelerate. Concurrently, for the same $C R$, the velocity profiles reveal an overshoot when the fluid flows through the contraction plane and enters the downstream duct (in accordance with the findings of Alves and Poole, 2007, Poole and Alves, 2009 and Sousa et al., 2009) particularly for the lower $C R$. The enhancement of the velocity undershoot and subsequent velocity overshoot in contraction flows of viscoelastic fluids was investigated by Alves and Poole (2007) for smooth planar contractions and shown to be pronounced at low contraction ratios, in agreement with our observation in a square-square contraction flow. In Fig. 12 we also include the numerical results obtained with the GNF model in order to demonstrate that the significant changes observed in the velocity profiles as De increases, as well as the onset of undershoots and overshoots in the centerline velocity profile are a consequence of elastic effects. For clarity, only the predicted velocity profiles for the higher flow rates are illustrated in Fig. 12 for each $C R$. The overshoot on the axial velocity profiles for the lower $C R$ is not observed in the numerical predictions using the GNF model, showing clearly that this is an effect of elasticity. The upstream shift of the velocity profile is also not captured in the GNF simulations. On the other hand, the GNF model is able to accurately predict the axial velocity in the downstream channel for fully developed flow conditions for $C R=8$. For $C R=2.4$ and 4 , due to the large velocity overshoots, the fully developed flow conditions downstream of the contraction plane were not achieved in the PIV measurements, but eventually the velocity profile will fall down to the values predicted by the GNF model. We note that the dimensionless velocity at the centerline under fully developed flow conditions is lower than that found for a Newtonian fluid, due to the shear-thinning rheological behavior of the GNF fluid.

Fig. 13 shows a contour plot of the velocity magnitude measured using PIV at the center plane of the square duct ( $y=0$ or $z=0$ ) for the flow of the viscoelastic fluid at $D e=76.3$ and $C R=2.4$. The velocity magnitude is scaled with the downstream average axial velocity, $U_{2}$. As can be seen, the velocity magnitude increases significantly near the contraction plane, which corresponds to the overshoot in the axial velocity profile at that location (cf. Fig. 12(a)). Moreover, we can also observe a large region with low velocities near the corners, a confirmation of the large recirculations observed at high $D e$ (cf. Fig. 5), and the existence of off-center maximum of the velocity in the upstream region, a consequence of divergent flow. 


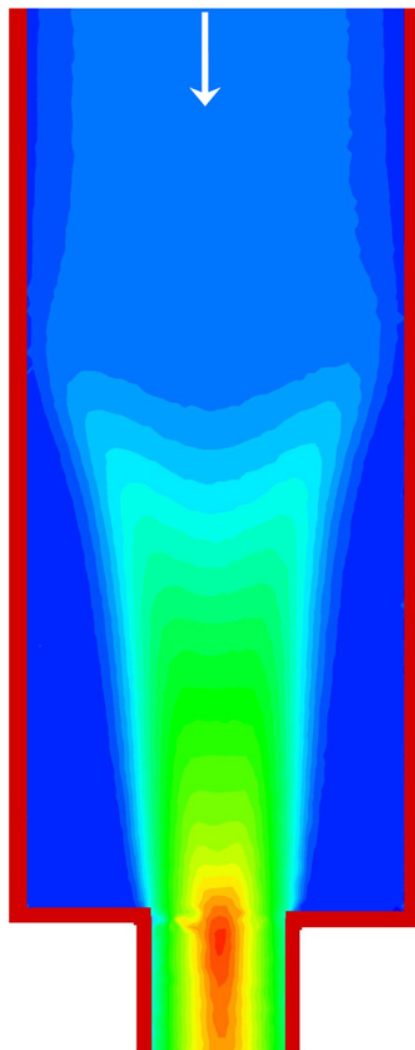

$\|\mathbf{u}\| / U_{2}$

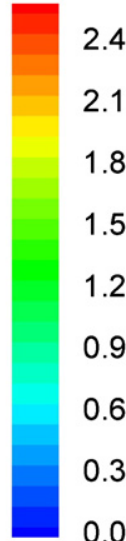

Fig. 13. Contour plot of the normalized velocity magnitude for the shear-thinning viscoelastic fluid measured with PIV at $D e=76.3, R e=0.670$ and $C R=2.4$.

\section{Conclusions}

The flow of a viscoelastic fluid with a shear-thinning rheological behavior, through square-square contractions was studied experimental and numerically and compared with the flow of a Newtonian fluid. In order to assess the effect of the contraction ratio on the fluid flow and with the purpose of establishing benchmark solutions of the non-Newtonian fluid flow through contractions with a square-square geometrical arrangement, different contraction ratios were investigated $(C R=2.4,4,8$ and 12$)$.

For the Newtonian fluid and for the range of contraction ratios studied, a vortex appears upstream of the contraction plane, which decreases in size with an increase of the flow inertia. For the viscoelastic fluid flow a complex 3D corner vortex also appears. At low Deborah numbers, the flow is similar to that found for the Newtonian fluid but when the flow rate (or the Deborah number) is increased, the vortex length increases significantly for all contraction ratios studied. Furthermore, when elastic effects are present, the increase of the vortex length is accompanied by a reversal of the flow direction within the 3D open vortical structure. For the lower contraction ratios, $C R=2.4$ and 4 , diverging streamlines appear when the Deborah number is increased. However, these diverging streamlines are not present in the flow through the 8:1 and 12:1 square-square contractions. Independently of $C R$, when elasticity is increased further, the flow eventually becomes time-dependent, with the size of the vortices varying in time.

\section{Acknowledgements}

The authors acknowledge the financial support provided by Fundação para a Ciência e a Tecnologia (FCT) and FEDER through projects PTDC/EME-MFE/70186/2006, PTDC/EQU-FTT/71800/2006, REEQ/262/EME/2005 and REEQ/928/EME/2005. P.C. Sousa would also like to thank FCT for financial support through scholarship SFRH/BD/ 28846/2006.

\section{References}

Afonso, A., Oliveira, P.J., Pinho, F.T., Alves, M.A., 2009. The log-conformation tensor approach in the finite-volume method framework. J. Non-Newt. Fluid Mech. 157, 55-65.

Alves, M.A., Oliveira, P.J., Pinho, F.T., 2003a. A convergent and universally bounded interpolation scheme for the treatment of advection. Int. J. Numer. Methods Fluids 41, 47-75.

Alves, M.A., Oliveira, P.J., Pinho, F.T., 2003b. Benchmark solutions for the flow of Oldroyd-B and PTT fluids in planar contractions. J. Non-Newt. Fluid Mech. 110, 45-75.

Alves, M.A., Oliveira, P.J., Pinho, F.T., 2004. On the effect of contraction ratio in viscoelastic flow through abrupt contractions. J. Non-Newt. Fluid Mech. 122 $117-130$

Alves, M.A., Pinho, F.T., Oliveira, P.J., 2005. Visualizations of Boger fluid flows in a 4:1 square-square contraction. AIChE J. 51, 2908-2922.

Alves, M.A., Pinho, F.T., Oliveira, P.J., 2008. Viscoelastic flow in a 3D square/square contraction: visualizations and simulations. J. Rheol. 52, 1347-1368.

Alves, M.A., Poole, R.J., 2007. Divergent flow in contractions. J. Non-Newt. Fluid Mech. 144, 140-148.

Bird, R.B., Armstrong, R.C., Hassager, O., 1987. Dynamics of Polymeric Liquids. Volume 1: Fluid Dynamics. John Wiley \& Sons, New York.

Boger, D.V., 1987. Viscoelastic flows through contractions. Ann. Rev. Fluid Mech. 19, 157-182.

Boger, D.V., Hur, D.U., Binnington, R.J., 1986. Further observations of elastic effects in tubular entry flows. J. Non-Newt. Fluid Mech. 20, 31-49.

Cable, P.J., Boger, D.V., 1978a. A comprehensive experimental investigation of tubular entry flow of viscoelastic fluids: Part I. Vortex characteristics in stable flow. AIChE J. 24, 868-879.

Cable, P.J., Boger, D.V., 1978b. A comprehensive experimental investigation of tubular entry flow of viscoelastic fluids: Part II. The velocity fields in stable flow. AIChE J. 24, 992-999.

Cable, P.J., Boger, D.V., 1979. A comprehensive experimental investigation of tubular entry flow of viscoelastic fluids: Part III. Unstable flow. AIChE J. 25, 152-159.

Chung, C., Kim, J.M., Hulsen, M.A., Ahn, K.H., Lee, S.J., 2009. Effect of viscoelasticity on drop dynamics in 5:1:5 contraction/expansion microchannel flow. Chem. Eng. Sci. 64, 4515-4524.

Dealy, J., Plazek, D., 2009. Time-temperature superposition-a users guide. Rheol Bull. 78, 16-31.

Denn, M.M., 2004. Fifty years of non-Newtonian fluid dynamics. AIChE J. 50, 2335-2345

Evans, R.E., Walters, K., 1986. Flow characteristics associated with abrupt changes in geometry in the case of highly elastic liquids. J. Non-Newt. Fluid Mech. 20 11-29.

Evans, R.E., Walters, K., 1989. Further remarks on the lip-vortex mechanism of vortex enhancement in planar contraction flows. J. Non-Newt. Fluid Mech. 32 95-105.

Evans, J.D., Sibley, D.N., 2008. Re-entrant corner flows of PTT fluids in the Cartesian stress basis. J. Non-Newt. Fluid Mech. 153, 12-24.

Fattal, R., Kupferman, R., 2004. Constitutive laws for the matrix-logarithm of the conformation tensor. J. Non-Newt. Fluid Mech. 123, 281-285.

Hulsen, M.A., Fattal, R., Kupferman, R., 2005. Flow of viscoelastic fluids past a cylinder at high Weissenberg number: stabilized simulations using matrix logarithms. J. Non-Newt. Fluid Mech. 127, 27-39.

Lu, Z., Khoo, B.C., Dou, H.-S., Phan-Thien, N., Yeo, K.S., 2006. Numerical simulation of fibre suspension flow through an axisymmetric contraction and expansion passages by Brownian configuration field method. Chem. Eng. Sci. 61, 4998-5009.

McKinley, G.H., Rodd, L.E., Oliveira, M.S.N., Cooper-White, J., 2007. Extensional flows of polymer solutions in microfluidic converging/diverging geometries. J. Cent South Univ. Technol. 14, 6-9.

Mompean, G., Deville, M., 1997. Unsteady finite volume simulation of Oldroyd-B fluid through a three-dimensional planar contraction. J. Non-Newt. Fluid Mech. 72, 253-279.

Nigen, S., Walters, K., 2002. Viscoelastic contraction flows: comparison of axisymmetric and planar configurations. J. Non-Newt. Fluid Mech. 102, 343-359.

Oliveira, M.S.N., Oliveira, P.J., Pinho, F.T., Alves, M.A., 2007. Effect of contraction ratio upon viscoelastic flow in contractions: the axisymmetric case. J. Non-Newt. Fluid Mech. 147, 92-108.

Oliveira, P.J., Pinho, F.T., Pinto, G.A., 1998. Numerical simulation of non-linear elastic flows collocated finite-volume method. J. Non-Newt. Fluid Mech. 79, 1-43.

Owens, R.G., Phillips, T.N., 2002. Computational Rheology. Imperial College Press, London.

Phan-Thien, N., Tanner, R.I., 1977. A new constitutive equation derived from network theory. J. Non-Newt. Fluid Mech. 2, 353-365.

Poole, R.J., Alves, M.A., 2009. Velocity overshoots in gradual contraction flows. J. Non-Newt. Fluid Mech. 160, 47-54.

Purnode, B., Crochet, M., 1996. Flows of polymer solutions through contractions Part 1: flows of polyacrylamide solutions through planar contractions. J. Non-Newt. Fluid Mech. 65, 269-289. 
Quinzani, L.M., Armstrong, R.C., Brown, R.A., 1995. Use of coupled birefringence and LDV studies of flow through a planar contraction to test constitutive equations for concentrated polymer solutions. J. Rheol. 39, 1201-1228.

Rothstein, J.P., McKinley, G.H., 1999. Extensional flow of a polystyrene Boger fluid through a 4:1:4 axisymmetric contraction-expansion. J. Non-Newt. Fluid Mech. 86, 61-88.

Sirakov, I., Ainser, A., Haouche, M., Guillet, J., 2005. Three-dimensional numerical simulation of viscoelastic contraction flows using the Pom-Pom differential constitutive model. J. Non-Newt. Fluid Mech. 126, 163-173.

Sousa, P.C., Coelho, P.M., Oliveira, M.S.N., Alves, M.A., 2009. Three-dimensional flow of Newtonian and Boger fluids in square-square contractions. J. Non-Newt. Fluid Mech. 160, 122-139.

Sousa, P.C., Coelho, P.M., Oliveira, M.S.N., Alves, M.A. Expansion flow in threedimensional square-square geometries. J. Non-Newt. Fluid Mech., submitted for publication.

van Doormal, J.P., Raithby, G.D., 1984. Enhancements of the SIMPLE method for predicting incompressible fluid flows. Numer. Heat. Transfer A 7, 147-163.
Walters, K., Webster, M.F., 1982. On dominating elastico-viscous response in some complex flows. Philos. Trans. R. Soc. London, Ser. A 308, 199-218.

Walters, K., 1985. Overview of Macroscopic Viscoelastic Flow. Viscoelasticity and Rheology. Academic Press, pp. 47-79.

Walters, K., Webster, M.F., Tamaddon-Jahromi, H.R., 2009. The numerical simulation of some contractionnext term flows of highly elastic liquids and their impact on the relevance of the Couette correction in extensional rheology. Chem. Eng. Sci. $64,4632-4639$.

White, S.A., Baird, D.G., 1988. Flow visualization and birefringence studies on planar entry flow behavior of polymer melts. J. Non-Newt. Fluid Mech. 29, 245-267.

Xue, S.-C., Phan-Thien, N., Tanner, R.I., 1998. Three dimensional numerical simulations of viscoelastic flows through planar contractions. J. Non-Newt. Fluid Mech. $74,195-245$.

Yue, O., Dooley, J., Feng, J.J., 2008. A general criterion for viscoelastic secondary flow in pipes of noncircular cross section. J. Rheol. 52, 315-332. 